\title{
A SURVEY FOR RR LYRAE VARIABLES IN THE LARGE MAGELLANIC CLOUD
}

\author{
NEILL REID \\ Palomar Observatory \\ Pasadena Ca. 91125 \\ $U S A$ \\ WENDY L. FREEDMAN \\ The Observatories of the Carnegie Institution of Washington \\ Pasadena Ca. 91101 \\ USA
}

\begin{abstract}
In this paper we report preliminary results from a survey of RR Lyrae variable stars in the Large Magellanic Cloud.
\end{abstract}

The RR Lyrae variables of the Large Magellanic Cloud (LMC) can be used as powerful probes both of the evolution of low-mass stars through the instability strip (the variation of luminosity with abundance and age and, in consequence, the reliability of these stars as distances indicators) and, using these stars as representatives of the older stellar populations in the LMC, of the kinematics and structure of the LMC during formation. However, with the exception of an outlying $\sim 1$ sq. deg. field (centred on NGC 2257) (Graham 1975, Nemec et al. 1985), most variable star surveys have concentrated on the clusters of the LMC. In this paper we report preliminary results from a survey that aims to remedy this defect by combining data from the du Pont 100-inch and the UK Schmidt telescopes.

The analysis described here is based on ten IIIaJ and one IIIaF UK Schmidt plates taken between January and March 1989 , and covering the $6^{\circ} \times 6^{\circ}$ field centred at $5^{h} 52^{m},-65^{\circ}$. These plates have been measured using the COSMOS facility at the Royal Observatory, Edinburgh, and we have positions, magnitudes, shapes and orientations for $\sim 1.1$ million images on each plate. Not all of these images are real, with spurious images contributed by diffraction spikes round brighter stars, diffuse gaseous emission, emulsion flaws and the like. Hence, as the first step in our analysis we have paired two $\mathrm{J}$ plates, retaining only matched images. The plates have a limiting magnitude of $B_{J} \geq 21.5$, significantly fainter than the magnitude range covered by $R R$ Lyraes, $\sim 18.6 \leq B_{J} \leq 20.0$, and so this procedure does not introduce any magnitude limit. This matched dataset of 974,017 stars forms the basis for subsequent plate-pairings.

We have transformed all the magnitudes onto the same instrumental system and standardised the instrumental scale using standard sequences from CCD observations of stars near H4 (Mateo \& Hodge 1986) and NGC 2155 (Mateo, priv. comm.). In doing so, we have adopted the relation

$$
B_{J}=B-0.32 \times(B-V)
$$


in transforming the B-band photometry onto the broader $B_{J}$ passband (Blair \& Gilmore 1982). We have applied no colour term to the R-band photometry.

In the plate-to-plate instrumental magnitude comparison, three of the ten $\mathrm{J}$ plates show a significantly larger dispersion about the mean transformation. In searching for candidate variables we have calculated the mean magnitude and $\mathrm{rms}$ dispersion about the mean for each object using only the seven better-quality plates. In addition, crowding affects a significant number of images with correspondingly inaccurate magnitudes. As in previous studies, we have allowed for this by omitting all images where the COSMOS best-fit ellipse has an axial ratio, $b / a, \geq 0.6$, and calculate $\left\langle B_{J}\right\rangle$ and $\sigma$ only when we have at least five good measurements. This reduces the sample size by $\sim 35 \%$.

We expect RR Lyraes to have a mean magnitude of $B_{J} \sim 19.5$, and at this magnitude the mean rms dispersion, $\sigma_{7}$, is $\sim 0.07 \mathrm{mag}$. Thus, as a first cut, we identify all stars with $\sigma_{7}>0.3$ as likely variables. From Monte Carlo simulations, where we take seven points at random from a light curve, we can show that this limit corresponds to a $50 \%$ probability of detecting a variable with a total amplitude of 0.9 mag - thus our sample is biased towards higher amplitude variables. This criterion gives a candidate list of 8,624 stars. However, in many cases the high $\sigma_{7}$ stems from one discrepant observation, and when we exclude these stars the final sample of variable-star candidates shrinks to 3610 stars, or $0.04 \%$ of the original sample.

We can also estimate the colours of these variables using our R photometry - although there are obvious uncertainties since we have only the one observation. Nonetheless, there are two clear groupings in the resulting colour-magnitude diagram, with approximately 900 objects clumped at $B_{J} \sim 20,\left(B_{J}-R\right) \sim 1.5$, at the tip of the red giant branch; these are likely to be semi-regular or long-period variables. Three hundred and sixty variables $(\sim 0.04 \%$ of the original sample) lie in the magnitude and colour range $18.5<B_{J}<20.0,-0.2<\left(B_{J}-\mathrm{R}\right)<0.7$. Given the uncertainties in the (B-R) colour, these are our candidate RR Lyraes. We have made a very preliminary attempt at estimating the periods for some of these stars and the variation is often at least consistent with their being RR Lyraes.

We intend to make spectroscopic observations of stars from the RR Lyrae sample using the du Pont telescope at Las Campanas in December, 1990. In addition, we hope to obtain both further Schmidt plates and CCD observations (with the Las Campanas 40-inch telescope) of individual stars to improve the accuracy of the period determinations.

\section{References}

Blair, M., Gilmore, G.F. (1982), Publ. Astron. Soc. Pac. 94, 742.

Graham, J.A. (1975), Publ. Astron. Soc. Pac. 87, 641.

Mateo, M., Hodge, P. (1986), Astrophys. J. Suppl. Ser. 60, 893.

Nemec, J.M., Hesser, J.E., Ugarte, P. (1985), Astrophys. J. Suppl. Ser. 57, 287. 